\title{
Analysis of quasi-optical power combiners by vector field measurements at $150 \mathrm{GHz}$
}

\author{
R. Judaschke ${ }^{1}$ and J. Weinzierl ${ }^{2}$ \\ ${ }^{1}$ Physikalisch-Technische Bundesanstalt, Bundesallee 100, 38116 Braunschweig, Deutschland \\ ${ }^{2}$ Universität Erlangen-Nürnberg Lehrstuhl für Hochfrequenztechnik, Cauerstraße 9, 91058 Erlangen, Deutschland
}

\begin{abstract}
A Two-dimensional quasi-optical power dividing/combining circuit has been experimentally investigated at $150 \mathrm{GHz}$. It consists of a rectangular horn antenna array as receiving/transmitting unit and a dual offset reflector setup to match the radiated field(s) to the pattern of the receiving antenna(s). To verify both design and adjustment of the quasi-optical circuit, electric field scans have been performed in selected planes and volumes of the setup. To measure the spatial electric field distribution, a vector field measurement system has been developed which operates in the frequency range between $148 \mathrm{GHz}$ and $152 \mathrm{GHz}$. Excellent agreement between calculated and measured results for a horn antenna array approve the predicted results calculated under the physical optics approximation. Measured power dividing/combining efficiency of the passive structure of $63 \%$ for an inter-element spacing of $10 \lambda$ indicates that the power combining principle is a suitable method up to submillimeter wavelengths.
\end{abstract}

\section{Introduction}

In the last years, significant efforts have been carried out to develop quasi-optical power combiners in order to overcome the basic limitations of solid-state devices on generating significant power levels at millimeter-wave frequencies (DeLisio et al., 2002; Harvey et al., 2000). To obtain both high power density and combining efficiency, the active devices (followed by radiating elements) are placed in a twodimensional array that radiates into an overmoded rectangular waveguide, coaxial waveguide, or quasi-optical resonator. Furthermore, quasi-optical setups have been investigated that use dielectric periodic structures and mirrors to couple the radiated power into a receiving antenna (Judaschke et al.,

Correspondence to: R. Judaschke

(rolf.judaschke@ptb.de)
2005). Such approaches are characterized by efficient and homogeneous power splitting/combining and solve the problem of heat dissipation due to array inter-element spacings $L_{x}$ of several wavelengths. However, their design and mechanical setup have to be carried out carefully in order to achieve both perfect field matching in the antenna aperture planes and, in case of amplifiers (Fig. 1), equal-in-phase and equal-in-power splitting/combining. To verify the quasioptical design, field mapping in magnitude and phase, performed in several circuit planes, is a suitable method, and furthermore, enables detection and elimination of mechanical misalignments.

In this paper, the power splitting/combining properties of a $5 \times 2$-element circuit is experimentally investigated by vector field measurements in the antenna array aperture plane and the input/output antenna aperture plane. In the first part, the field measurement system, including field scanning probe, flexible waveguides, signal generation, and IF-processing is described. In the second part, experimental results for the power splitting/combining circuit at $150 \mathrm{GHz}$, as well as comparisons with theoretical results are given.

\section{$2150 \mathrm{GHz}$ field measurement system}

To measure the spatial electric field distribution in a quasioptical circuit, a vector measurement system has been developed, the general setup of which is shown in Fig. 2. It operates at frequencies between $148 \mathrm{GHz}$ and $152 \mathrm{GHz}$ and consists of a heterodyne receiver that uses two channels, a signal channel mounted on a 3D scanning platform and a spatially fixed reference channel which is necessary for phase detection. The RF signals are received by a pair of dielectric rod antennas (field probes) and fed to the mixer ports via flexible dielectric waveguides. In order to maximize the overall system sensitivity, fundamental balanced mixers are used in both receiver channels which convert the received RF sig-

Published by Copernicus Publications on behalf of the URSI Landesausschuss in der Bundesrepublik Deutschland e.V. 


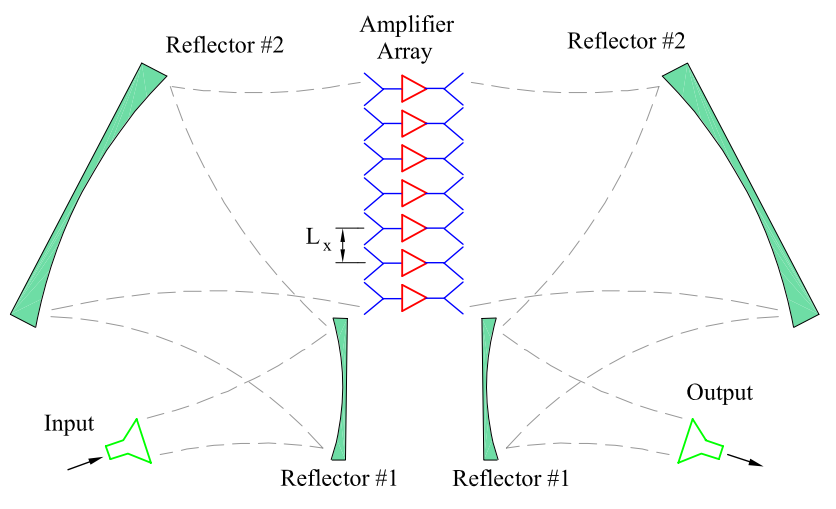

Fig. 1. Quasi-optical amplifier with symmetric power splitting/combining circuitry.

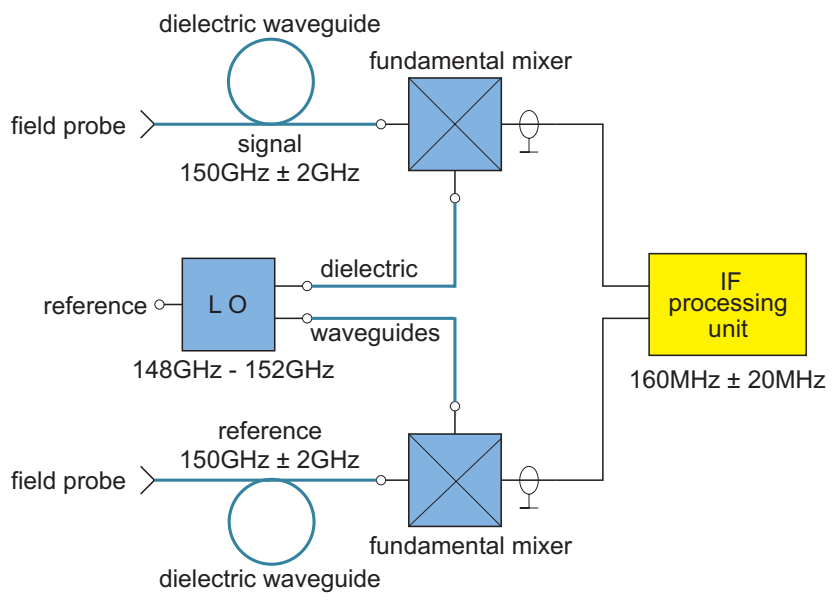

Fig. 2. System concept of the vector field measurement system.

nals directly down to the IF frequency with a considerably low conversion loss of less than $9 \mathrm{~dB}$. The IF-detection and processing is performed at $160 \mathrm{MHz}$ with considerably enhanced measurement accuracy by implementing a software correction algorithm. The system is operated by a PC which controls mechanical movements, data acquisition, real-time data correction and graphical user interface. The system shows a dynamic range of $75 \mathrm{~dB}$ and an overall phase uncertainty of less than $2^{\circ}$.

\subsection{Dielectric waveguides}

Due to the relative motion between the field-scanning probe on the one hand and the fixed reference channel as well as the mixers on the other hand, the RF signals are guided to the mixers via flexible dielectric waveguides of rectangular cross section, made of polyethylene $\left(\epsilon_{r}=2.3\right)$. The design of the dielectric waveguides is based on numerical field simulations which include design and optimization of waveguide cross section, matched transition to standard metallic rectangular (a)

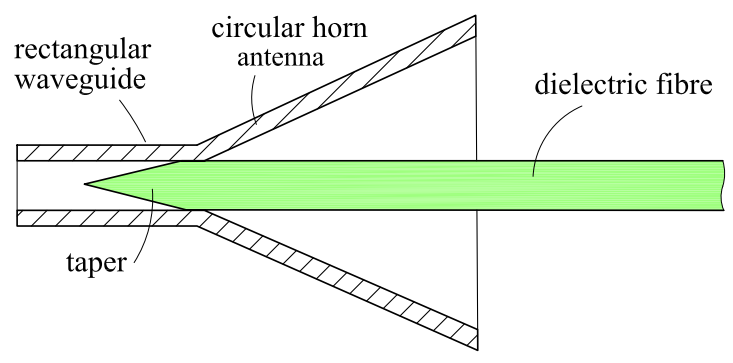

(b)

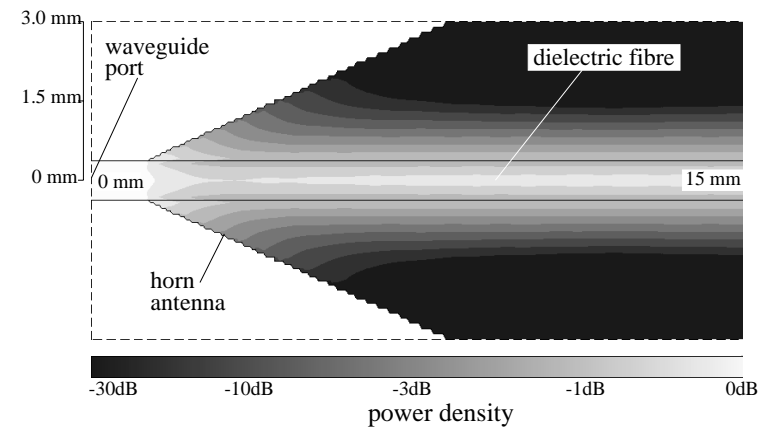

Fig. 3. (a) Cross section of waveguide-to-fibre transition, (b) power density around the dielectric waveguide at $150 \mathrm{GHz}$ (numerical simulation).

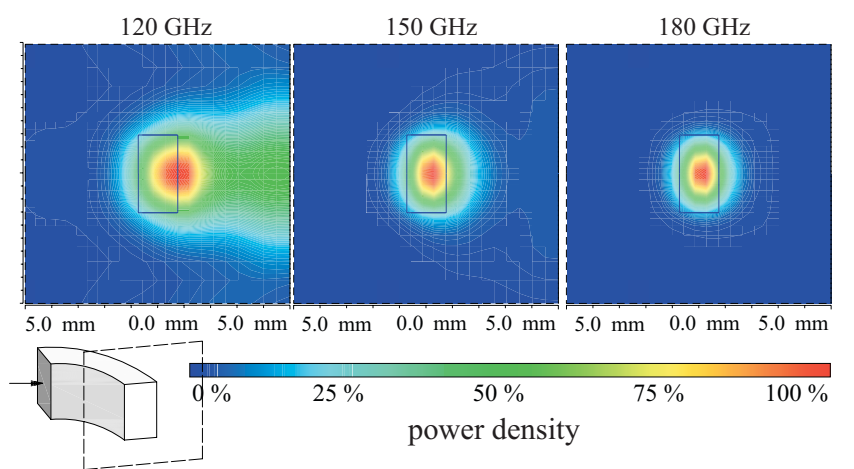

Fig. 4. Cross-sectional power density of a bended rectangular dielectric fibre, bending radius $\mathrm{r}=15 \mathrm{~mm}$.

waveguides, and investigation of bending properties.

Figure 3a illustrates the cross section of the waveguideto-fibre transition. It turns out that an optimized transition for low loss, broadband operation, polarization conservation, and minimum reflection consists of a fibre of rectangular cross section equal in size with respect to the metallic waveguide dimensions (WR-6, $1.65 \times 0.825 \mathrm{~mm}^{2}$ ), a tip taper inside the waveguide transition, and an optimum angle of the circular transition horn of $38^{\circ}$. Figure $3 \mathrm{~b}$ shows the simulated power density distribution across the transition along the dielectric fibre. The fraction of power inside the fibre is frequency-dependent and amounts to approx. $80 \%$ at $150 \mathrm{GHz}$ in the homogeneous region. This property requires adequate fibre assembling techniques, i.e. supporting the fibre by rigid expanded foam. Additionally, the simulations 


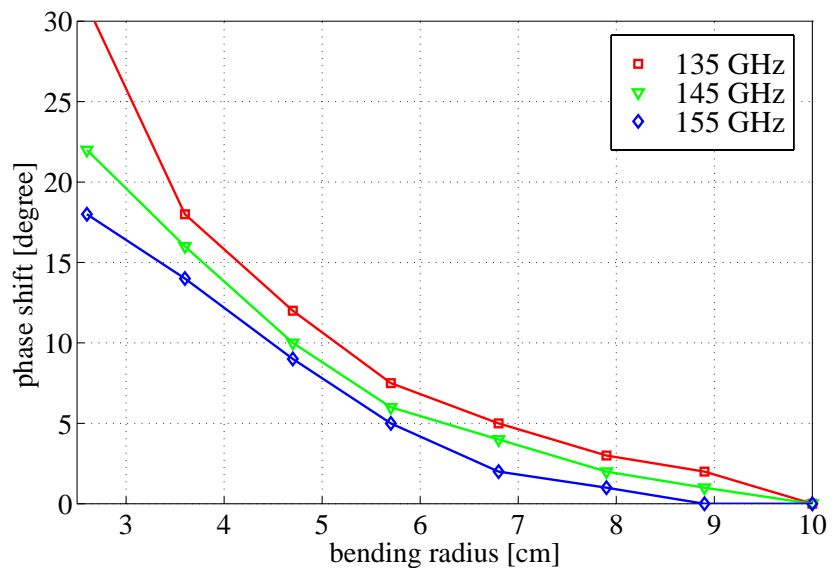

Fig. 5. Measured phase shift of bended dielectric fibres.



Fig. 6. Measured attenuation of dielectric waveguides at $150 \mathrm{GHz}$.

provide information about the phase shift in case of a bended fibre. In Fig. 4, the cross-sectional power density of a bended fibre is shown for three different operating frequencies.

Obviously, the waveguiding of the fibre improves with increasing frequency, and the power density maximum is shifted towards the fibre core. This property affects the electrical length of the fibre and hence, results in an additional phase shift.

As shown in Fig. 5, the measured additional phase shift as a function of the bending radius gives information about the phase uncertainty in case of a moving scanning unit. It is significant, that the phase shift becomes significant for bending radii smaller than $10 \mathrm{~cm}$ which has to be taken into account for accurate phase measurements.

Figure 6 compares attenuation measurements of the dielectric waveguide in comparison with a metallic D-band waveguide.

\subsection{Field probe}

To minimize the field distortions caused by the measurement system, dielectric antennas are used as field probes which are formed by tapered fibre endings. Especially the taper geom-

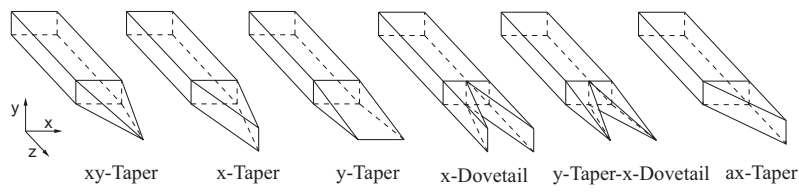

Fig. 7. Investgated tip types of the dielectric field probe.

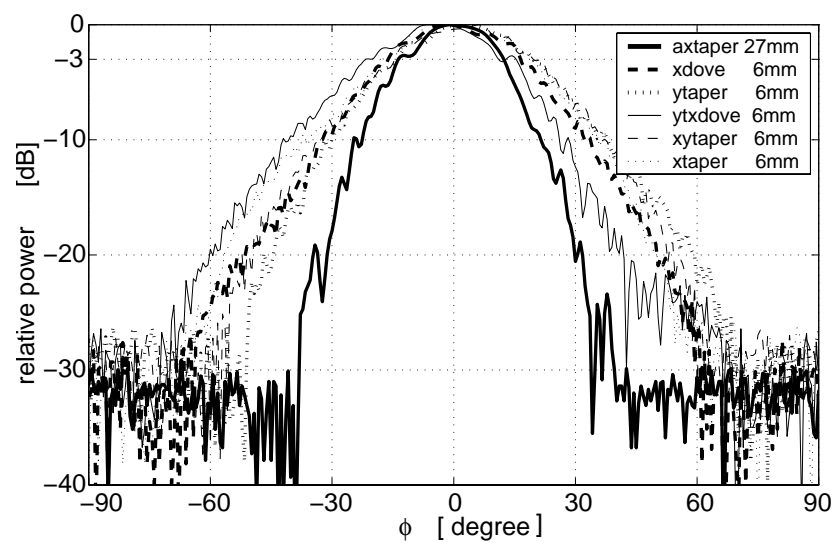

Fig. 8. Measured horizontal radiation pattern for different tip geometries and lengths.

etry was investigated by 3D field simulations and extensive measurements. It turned out that a decrease of tip width results in a smaller beamwidth especially in the horizontal antenna radiation pattern. Due to ease of fabrication, a long asymmetrical ax-taper (tip length: $27 \mathrm{~mm}$ ) has been chosen, the measured half power beamwidth of which is less than $25^{\circ}$ (cp. Fig. 7). The measured polarization decoupling of this antenna geometry is better than $30 \mathrm{~dB}$.

The field probe has approximately a Gaussian beam profile with asymmetric beam waists $w_{x} \approx 2.0 \mathrm{~mm}$ and $w_{y} \approx$ $1.5 \mathrm{~mm}$ at $150 \mathrm{GHz}$. Due to the characteristic of the field scanning probe, the actual electric field $E_{a}(x, y)$ at point $(x, y)$ is weighted by the electric field $E_{p}$ of the field probe to get the measured field $E_{m}(x, y)$ (Höft et al., 2002) according to

$$
\begin{aligned}
& E_{m}(x, y)= \\
& \int_{-\infty}^{\infty} \int_{-\infty}^{\infty} \bar{E}_{p}\left(k_{x}, k_{y}\right) \bar{E}_{a}{ }^{*}\left(k_{x}, k_{y}\right) d k_{x} d k_{y} \\
& E_{a}(x, y) \cdot \frac{-\infty-\infty}{4 \pi^{2} \cdot C_{n, p} \cdot C_{n, a}}
\end{aligned}
$$

with normalization

$$
C_{n}=\sqrt{\int_{-\infty}^{\infty} \int_{-\infty}^{\infty} E(\xi, \eta) E^{*}(\xi, \eta) d \xi d \eta}
$$

and Fourier transformation

$$
\bar{E}\left(k_{x}, k_{y}\right)=\int_{-\infty}^{\infty} \int_{-\infty}^{\infty} E(x, y) e^{j\left(x k_{x}+y k_{y}\right)} d x d y .
$$




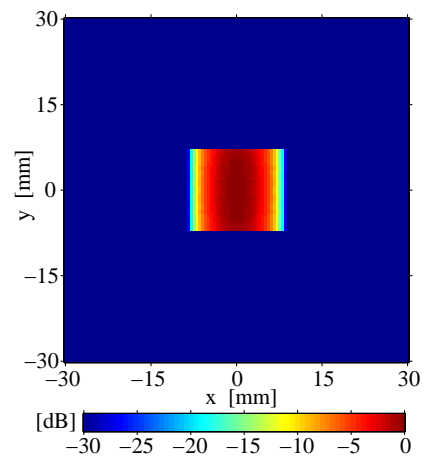

(a)

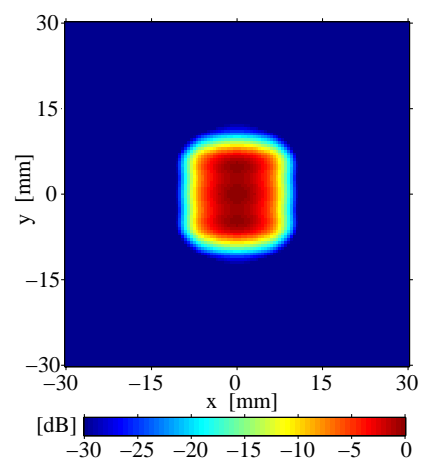

(b)
Fig. 9. Aperture field of a rectangular horn antenna: (a) magnitude of electric field $E_{y}$ calculated with GSM method, (b) theoretical aperture field from (a) weighted with ax-taper field probe characteristic.

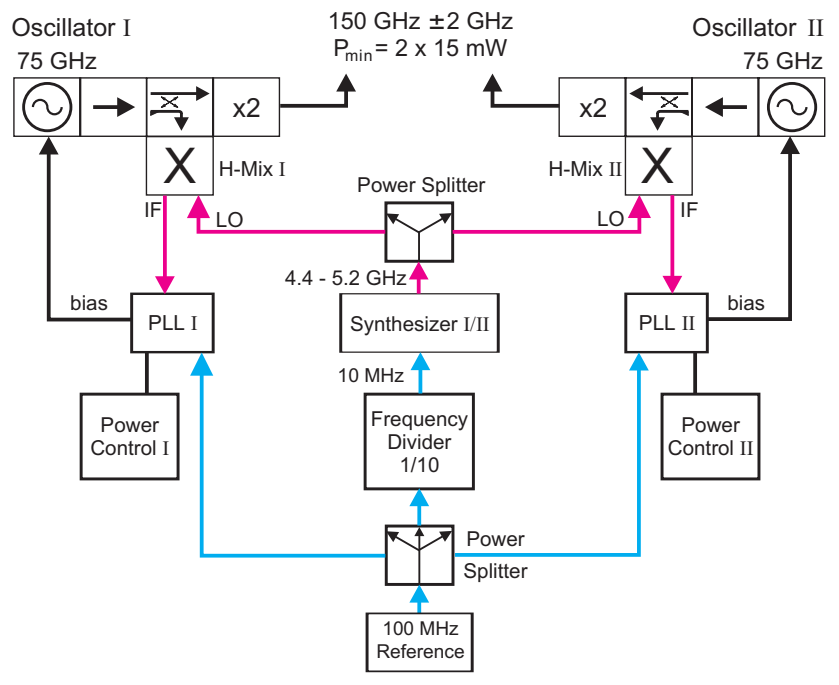

Fig. 10. $150 \mathrm{GHz}$ local oscillator unit.

Equation (1) constitutes the tradeoff between high sensitivity and selectivity on the one hand - realized by a narrow probe beamwidth to receive the signal exclusively from the expected direction of wave incidence - and an isotropic probe to detect all field contributions at the probe location. Figure 9 illustrates the effect of limited selectivity of the used ax-taper probe: scanning the aperture field $E_{y}$ of a rectangular horn antenna (theoretical field calculated with generalized scattering parameter (GSM) method in Fig. 9a) would give the diffuse image shown in Fig. 9b. As expected, the measurement error is going to be most significant at field discontinuities.

\subsection{Receiver unit}

The fundamental mixers (cp. Fig. 2) are pumped by a solid state local oscillator unit which is tunable from $148 \mathrm{GHz}$ to $152 \mathrm{GHz}$, corresponding to a measurement bandwidth of $150 \pm 2 \mathrm{GHz}$. Due to the fundamental mixer LO power requirement of $8 \mathrm{~mW}$ and feeding losses of $3 \mathrm{~dB}$, a minimum

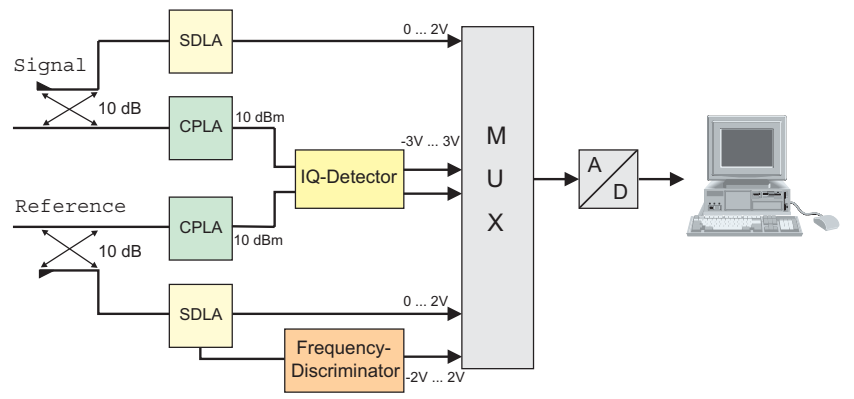

Fig. 11. IF processing unit.



Fig. 12. Real-time correction algorithm for the IF unit.

output power of $2 \times 15 \mathrm{~mW}$ is generated by a pair of GUNN oscillators at $75 \mathrm{GHz}$ followed by frequency doublers as shown in Fig. 10. Both GUNN oscillators are PLL-locked on a $100 \mathrm{MHz}$-reference oscillator and one common synthesizer which is essential for phase measurements.

The synthesizer is controlled by a computer via a parallel interface which allows the output frequency to be set in steps of $2 \mathrm{MHz}$ over a range of $400 \mathrm{MHz}$ without manually readjusting the PLL-module control voltage. The vector analysis of the measured signals is processed in the IF frequency range at $160 \mathrm{MHz}$. Figure 11 shows the block diagram of the IF processing unit.

In both channels, the received signal is split up by $10 \mathrm{~dB}$ couplers. The minor part of the signal is amplified by successive detection logarithmic amplifiers (SDLA). Their video outputs are indicators for the signal power in each channel. The major part of the signals is used for phase difference measurements between the channels. It is amplified by constant phase limiting amplifiers (CPLA) in order to eliminate the influence of signal power variations during field scanning. These limiters work properly in the power range from $-65 \mathrm{dBm}$ to $+10 \mathrm{dBm}$ which limits the dynamic range of the whole IF processing unit. The limited signals (power level $10 \mathrm{dBm}$ ) of both channels are processed by an IQ-detector (bandwidth $40 \mathrm{MHz}$ ) which generates video output signals proportional to the in-phase and to the quadrature component of the channel phase difference.

The video signal post processing is performed by a personal computer which is connected to the IF unit via interface 


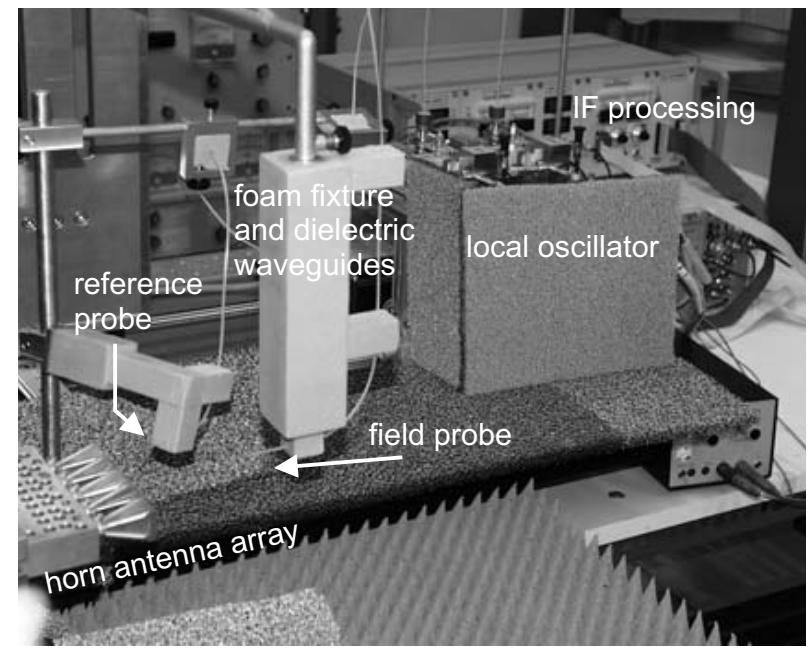

Fig. 13. Measurement of a 5 element linear horn antenna array.

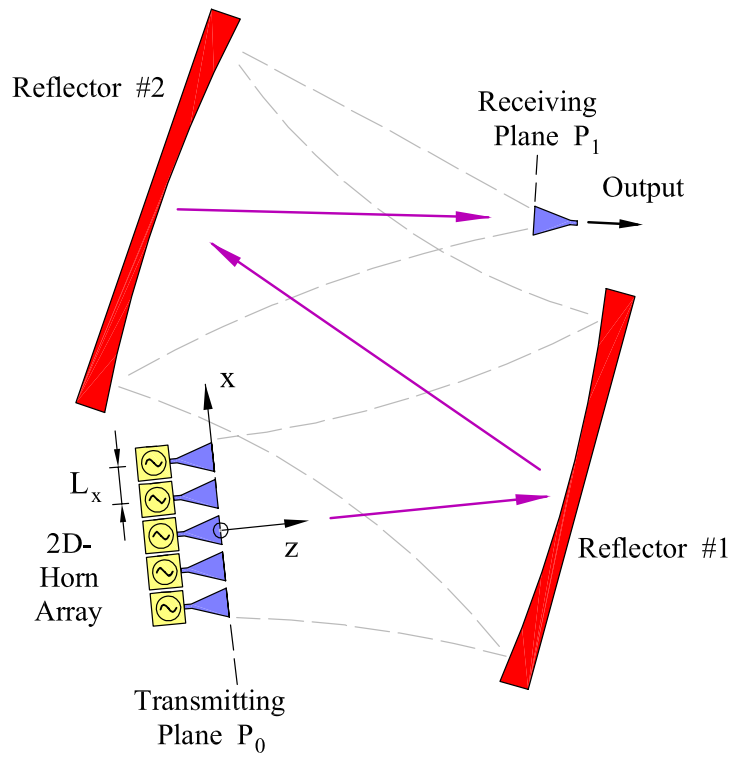

Fig. 14. Power combiner with two-dimensional horn array, shaped reflectors, and receiving rectangular horn antenna.

card, 16-bit A/D-converter, and analog multiplexer. Since the characteristic of all IF components is dependent on both frequency and power, a real-time software correction was implemented, leading to a significant improvement of measurement accuracy. Assuming that the phase uncertainty of the whole measurement system was specified to be less than $3^{\circ}$, the phase response of about $4^{\circ}$ in each limiting amplifier has to be taken into account. The required input power information is provided by logarithmic detection amplifiers (SDLA), the frequency response of which again is corrected using a calibrated frequency discriminator in the reference channel.

The moving field probe is mounted on a precise stepper motor controlled 3-axis scanning unit supported by precision bearings to achieve a spatial resolution of $6.25 \mu \mathrm{m}$ which is corresponding to a single stepper motor increment. The mea- (a)

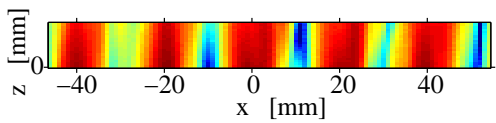

(b)
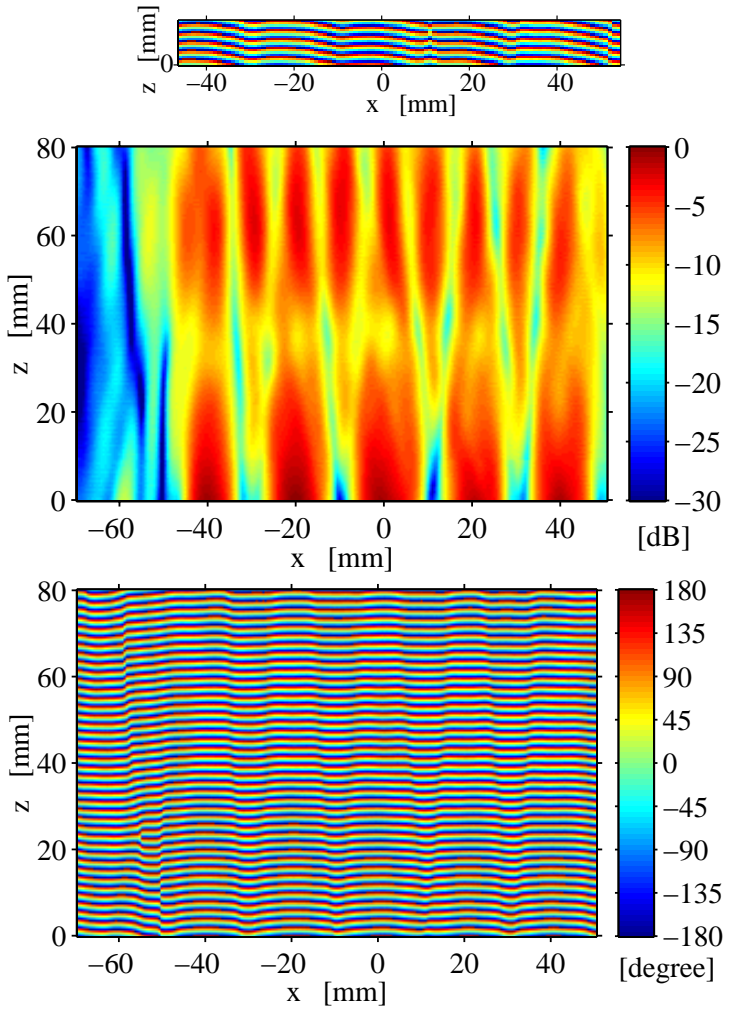

Fig. 15. Horizontal field scan in front of the $5 \times 2$ horn array at $y=+10 \mathrm{~mm}$ : (a) magnitude and (b) phase of the misaligned setup, (c) magnitude and (d) phase of the mechanically aligned setup.

sured reproducibility of an arbitrary position is \pm 1 step, allowing high precision 3D scanned field measurements. The fixtures for both field probes are made of an expanded foam material to avoid both distortions of the guided waves on the dielectric fibres as well as reflections of the propagating waves in the measurement volume during field scanning. Figure 13 shows the scanning unit in front of a $5 \times 1$ element linear horn array.

\section{Quasi-optical power combiner}

In comparison to the power combining scheme reported in (Judaschke et al., 2005) where a periodic, dielectric grating has been placed in front of the horn array, the $5 \times 2$ element circuit under investigation consists of two parabolically shaped mirrors having an arbitrary, computer-generated profile superimposed on their surface, as shown in Fig. 14. The array inter-element spacings were chosen to be identical $L_{x}=L_{y}=10 \lambda$ in both directions, and are given by the horn aperture width. The distance between the array aperture and the center of reflector \#1 amounts to $90 \lambda$, the distance between the mirror centers $130 \lambda$, respectively, which produces 
(a)

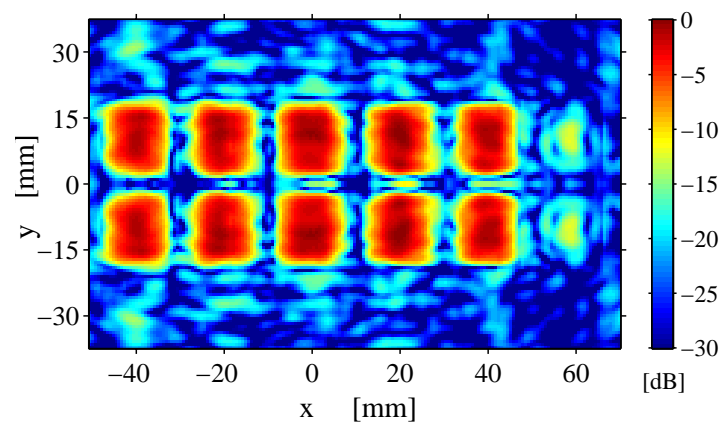

(b)

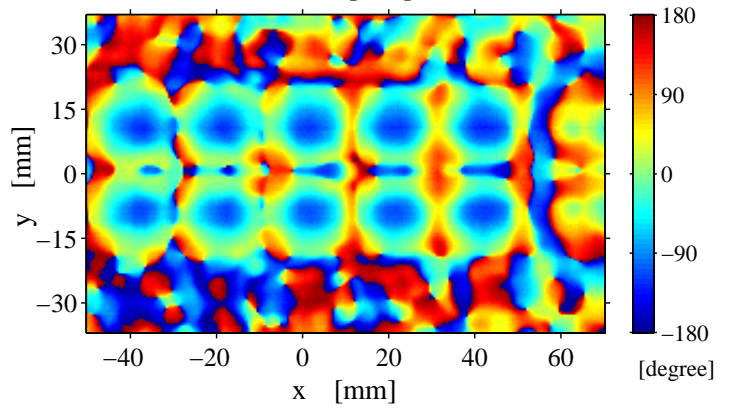

(c)

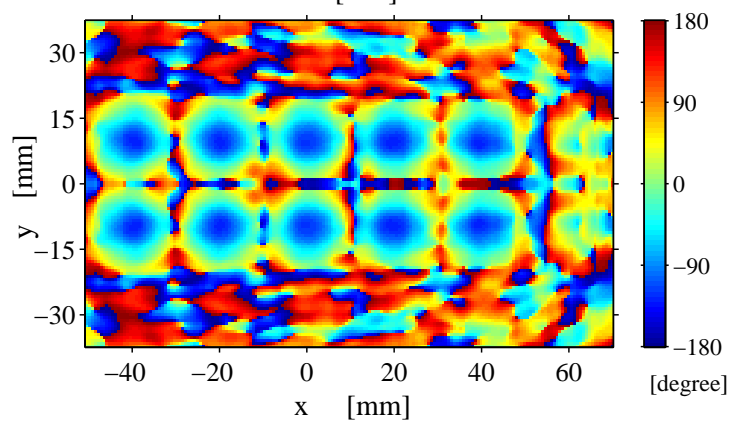

(d)

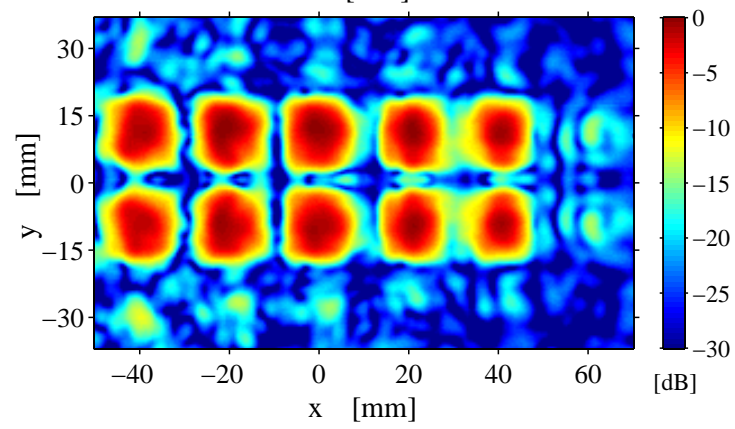

Fig. 16. Calculated (a) and measured (b) magnitude, calculated (c) and measured (d) phase of electric field $E_{y}$ of $5 \times 2$ power splitter in transmitting plane.

efficient reflector illumination under the given array size and horn antenna geometry.

The reflectors in dual-offset configuration are designed by calculating the surface magnetic fields under the physical optics approximation and applying a gradient method (Judaschke et al., 2006) followed by a smoothing procedure to achieve manufacturable surfaces (radius $\leq 6 \mathrm{~mm}$ ).

The reflector surfaces were allocated to matrices of size $360 \times 360$ each, which amounts to 259200 variables to be determined within the optimization.

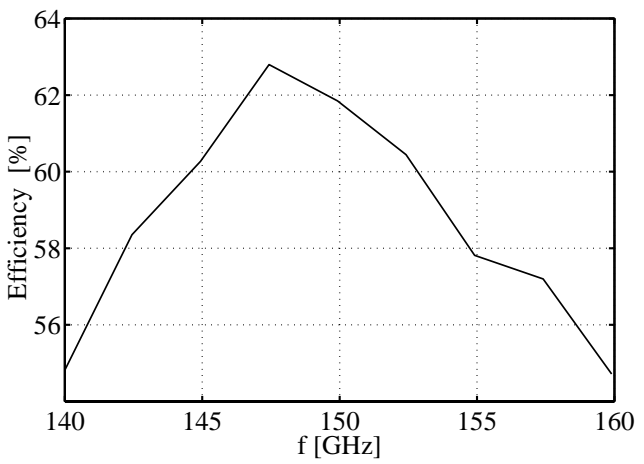

Fig. 17. Overall power splitting efficiency.
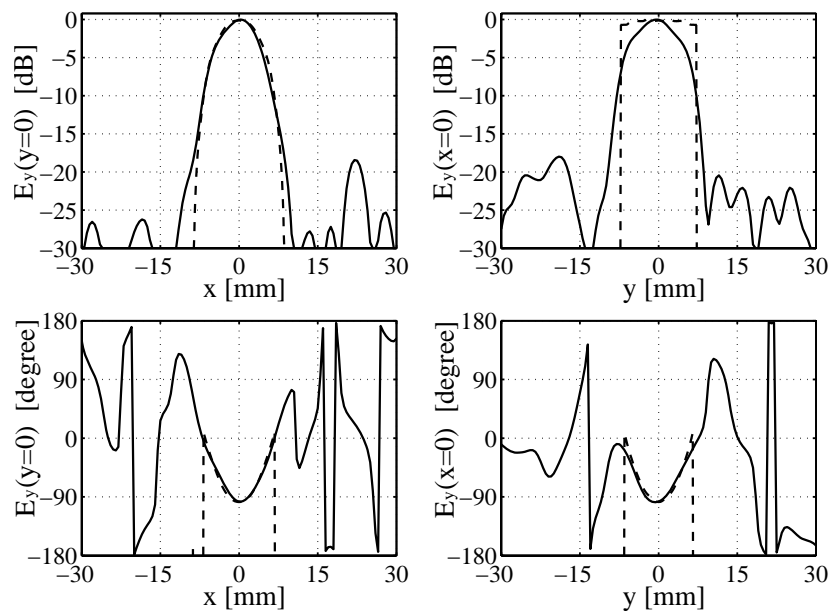

Fig. 18. Normalized magnitude and phase of electric field $E_{y}$ in receiving plane $P_{1}$ : superposition of $5 \times 2$ measured fields for singleport excitation in plane $P_{0},(-)$ measurement and (----) ideal case.

In a first step, the quasi-optical design has been investigated as a power splitter by scanning the electric field distribution both in horizontal planes in front of the horn array and in the transmitting plane $P_{0}$. For these measurements, the horn array has to be removed, and hence, standing wave effects due to reflections at the array aperture cannot be measured. The combiner has been designed for in-phase operation of the active elements, as required in case of a quasioptical amplifier (cp. Fig. 1). As shown in Fig. 15, possible phase imbalances due to mechanical misalignments can be detected by horizontal field scans (inclined wave incidence in Fig. 15b).

Figure 16 compares theoretical and measured field $E_{y}$ in plane $P_{0}$ which are in excellent agreement for both amplitude and phase. The phase variation between the antenna spots was determined to be less than $\pm 18^{\circ}$ which is close to the design. With the horn array assembled, the overall power splitting efficiency is calculated by adding up the measured output power contributions at the $5 \times 2$ array ports with respect to the input power at the receiving horn. Broadband 


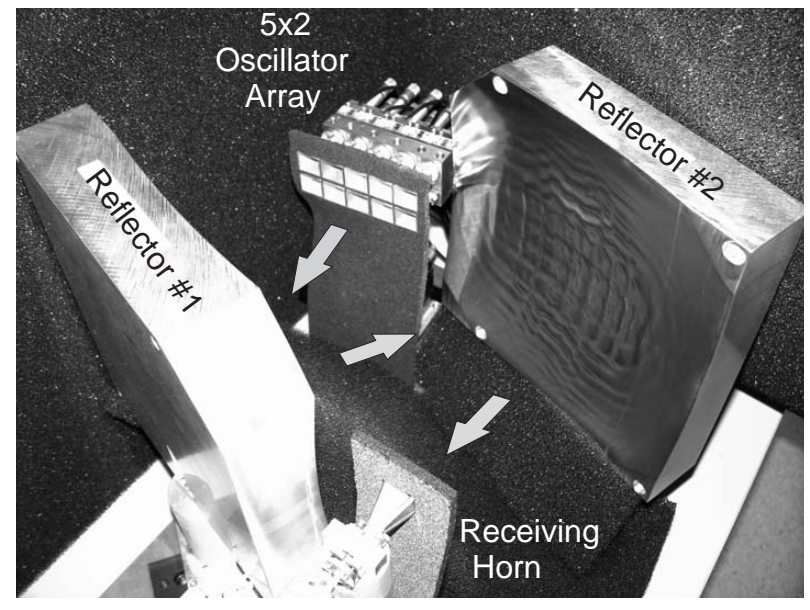

Fig. 19. Photograph of $5 \times 2$ quasi-optical power combiner.

behavior with a maximum efficiency of $63 \%$ at $148 \mathrm{GHz}$ is depicted in Fig. 17.

To investigate the passive circuit in power combining operation, $5 \times 2$ field scans were performed in receiving plane $P_{1}$ with the array ports successively excited by a phase-locked oscillator.

Figure 18 shows the superimposed field which is in close coincidence with the theoretical aperture field in H-plane. However, the measured field gradient towards the horn boundaries in E-plane as compared with the theoretically approx. constant electric field indicates mismatch which limits the overall efficiency. However, by using a dual mode or corrugated horn instead, field matching and subsequently increased efficiency can be expected.

Finally, the array was excited by tunable IMPATT oscillators. Due to mismatch in plane $P_{1}$ mutual oscillator coupling results in synchronized operation. By tuning the free-running frequencies of the individual oscillators, phase imbalances of the oscillating ensemble could be eliminated which gave a maximum power combining efficiency of $61.4 \%$.

\section{Conclusions}

A vector field measurement system has been described which operates in the frequency range between $148 \mathrm{GHz}$ and $152 \mathrm{GHz}$. To measure the spatial electric field distribution in both magnitude and phase, a dual channel heterodyne receiver detects the signals received from a moving and a fixed field probe, respectively. The system has a dynamic range of $75 \mathrm{~dB}$ and an overall phase uncertainty of less than $2^{\circ}$. The system has been used to investigate a two-dimensional quasi-optical power splitter/combiner at $150 \mathrm{GHz}$. The combiner consists of a $5 \times 2$ element rectangular horn array, a dual-offset reflector setup, and a receiving rectangular horn antenna. Field scans in both receiving and plane of the combiner provide insight into the spatial field distribution of the quasi-optical circuit and enable the detection and elimination of mechanical misalignments. An overall power combining efficiency of $61.4 \%$ at $150 \mathrm{GHz}$ has been measured for the active circuit excited by $5 \times 2$ IMPATT oscillators.

Acknowledgements. The authors are indebted to the Deutsche Forschungsgemeinschaft for financial support.

\section{References}

Chatterjee, R.: Dielectric and Dielectric-Loaded Antennas, Research Studies Press RSP, 1985.

DeLisio, M. P. and York, R. A.: Quasi-optical and spatial power combining, IEEE Trans. Microwave Theory \& Tech., 12, 929936, 2002.

Harvey, J., Brown, E. R., Rutledge, D. B., and York, R. A.: Spatial power combining for high-power transmitters, IEEE Microwave, $1,48-59,2000$.

Hofmann, A., Hörster, E., Weinzierl, J., Schmidt, L.-P., and Brand, H.: Flexible Low-Loss Dielectric Waveguides for THz Frequencies with Transitions to Metal Waveguides, in: Proceedings of the 33th European Microwave Week, Munich, Germany, 2003.

Hofmann, A., Seibert, G., Manglberger, M., Kalb, A., Göbel, A., Yao, S., Weinzierl, J., Schmidt, L.-P., and Brand, H.: A Modular Vector Field Measurement System at $150 \mathrm{GHz}, 300 \mathrm{GHz}$ and $450 \mathrm{GHz}$, in: Proceedings of the German Microwave Conference 2005, Ulm, Germany, 2005.

Höft, M., Weinzierl, J., and Judaschke, R.: Broadband analysis of holographic power combining circuits, Int. Journal of Infrared and Millimeter Waves, 23, 1127-1146, 2003.

Judaschke, R., Höft, M., and Schünemann, K.: Quasi-optical $150 \mathrm{GHz}$ power combining oscillator, IEEE Microwave and Wireless Components Letters, 15, 300-302, 2005.

Judaschke, R., Magath, T., and Schünemann, K.: 2-D Quasi-optical power combining oscillator array at D-band, Microwave Symposium Digest, IEEE MTT-S International, San Francisco, USA, 634-637, 2006.

Weinzierl, J., Fluhrer, Ch., and Brand, H.: Dielectric Waveguides at Submillimeter Wavelengths, in: IEEE Sixth International Conference on THz Electronics, Leeds, UK, 1998.

Weinzierl, J., Richter, J., Brand, H., and Schmidt, L.-P.: Simulation and Measurement of Dielectric Antennas at $150 \mathrm{GHz}$, in: Proceedings of the 29th European Microwave Week, 2, 185-188, Munich, Germany, 1999.

Weinzierl, J., Schulz, R., Brand, H., and Schmidt, L.-P.:: A Vector Field Measurement System for $150 \mathrm{GHz}$, in: Proceedings of 25th Conference on Infrared and Millimeter Waves (IRMMW 2000), Peking, V.R. China, 2000.

Weinzierl, J., Brand, H., and Schmidt, L.-P.: Vector Field Measurements at $150 \mathrm{GHz}$, in: Proceedings of the 11th Conference and Exhibition on Microwaves, Radio Communication and Electromagnetic Compatibility MIOP 2001, Stuttgart, Germany, 2001. 